\title{
SELF-ORGANIZED NANOPATTERNS INDUCED BY ION MILLING OF THIN FILMS
} Qiangmin Wei*, Sha Zhu ${ }^{* *}$, Lumin Wang,**

*Department of Materials Science and Engineering, ${ }^{* *}$ Department of Nuclear Engineering \& Radiological Sciences, University of Michigan, Ann Arbor, MI 48109, USA.

The bombardment of solid surfaces with low energetic ions is the focus of intensive investigations due to its possibility of inducing well-ordered periodic structures via self-assembly. A variety of topographies can result from surface erosion depending on sputtering conditions and material properties. Ripples, bumps, cones and fingerprint-like structures are the typical morphologies with the wavelength scales in the order of $10-1000 \mathrm{~nm}^{1-3}$. Here we demonstrate that a variety of intriguing patterns can be induced by argon ions of several $\mathrm{keV}$ on ultrathin polymer films at glancing angle of incidence with the simultaneous sample rotation. These periodic nanostructures on films can be used as the templates to fabricate densely packed nanometer-scale structures by deposition of atoms. The experiments were carried out in a Gatan Precision Ion Polishing System (PIPS) with an argon beam of $2-5 \mathrm{keV}$ under a pressure of $3 \times 10^{-3}$ torr. The incident angle of the $\mathrm{Ar}^{+}$ions was $80^{\circ}$ from the surface normal. Typical ion flux used in the experiments was $10^{14}$ ions $/ \mathrm{cm}^{2} \mathrm{~s}$.

The TEM images of a polymer film made of a cured Gatan M-bond 610 epoxy resin before and after argon ion irradiation with various ion fluences at a constant speed of specimen rotation are shown in Fig.1. After an ion fluence of $10^{17}$ ions $/ \mathrm{cm}^{2}$, the sand dune like ripples with a wavelength of $25 \mathrm{~nm}$ appeared (Fig. 1B). With the increase of exposure time, the polymer films evolve into striped patterns (Fig.1C), and the stripes tend to become wider with the increasing ion fluence (Fig. 1D). Fig 1E shows a curved ripple structure in an area of the sample shown in Fig. 1D. Fig. 1F is another pattern with the wavelength of only $7 \mathrm{~nm}$. It can be seen that these stripes consist of defects similar to vacancies clusters lining up along the stripes which ccould also be found in Fig.1C. Fig. 2 illustrates the nanopattens made of sputtered (from the adjacent copper grid) and redeposited copper atoms. Uniformly sized and shaped copper nanoparticles formed ordered arrays of stripe-like nanostructure on the thin polymer film made of the epoxy resin. Figs. 2C and 2D are the HRTEM image of Figs. 2A and $2 \mathrm{~B}$, respectively, illustrating the crystalline nature of the copper nanodots and nanostripes.

It can be seen that polymers irradiated by low energy ions underwent degradation during ion bombardment in which chain scission, aggregation, and molecular emission occurred (Figs.1 B and C). These effects are due to the large amount of energy storage in the molecular environment, which overcomes the binding energies of simple organic molecules. At high fluence, the nucleation and growth of clusters of amorphous carbon and radicals were generated, leading to the final structure that has little memory of original chain structure. Two opposite effects were resulted from the irradiation of polymers: one is related to crosslinking or chemically joining of two or more molecules by a covalent bond and the second to a chain scission or the breaking of a molecular bond and shortening of the overall chain, corresponding to the roughening and smoothing on the irradiated surface of bulk materials respectively[2]. These effects induced by energetic ions on the surface of the polymer film can drive the system far from the equilibrium, that finally contributes to the pattern formation [2].

[1] R.M. Bradley, and J.M.E. Harper, 6 (1988) 2390-2395.

[2] S. Facsko, T. Dekorsy, C. Koerdt, C. Trappe, H. Kurz, A. Vogt, and H.L. Hartnagel, Science 285 (1999) 1551-1553.

[3] F. Frost, A. Schindler, and F. Bigl, Physical Review Letters 85 (2000) 4116-4119.

[4] Work supported by US DOE under grant DF02-02ER46005. 

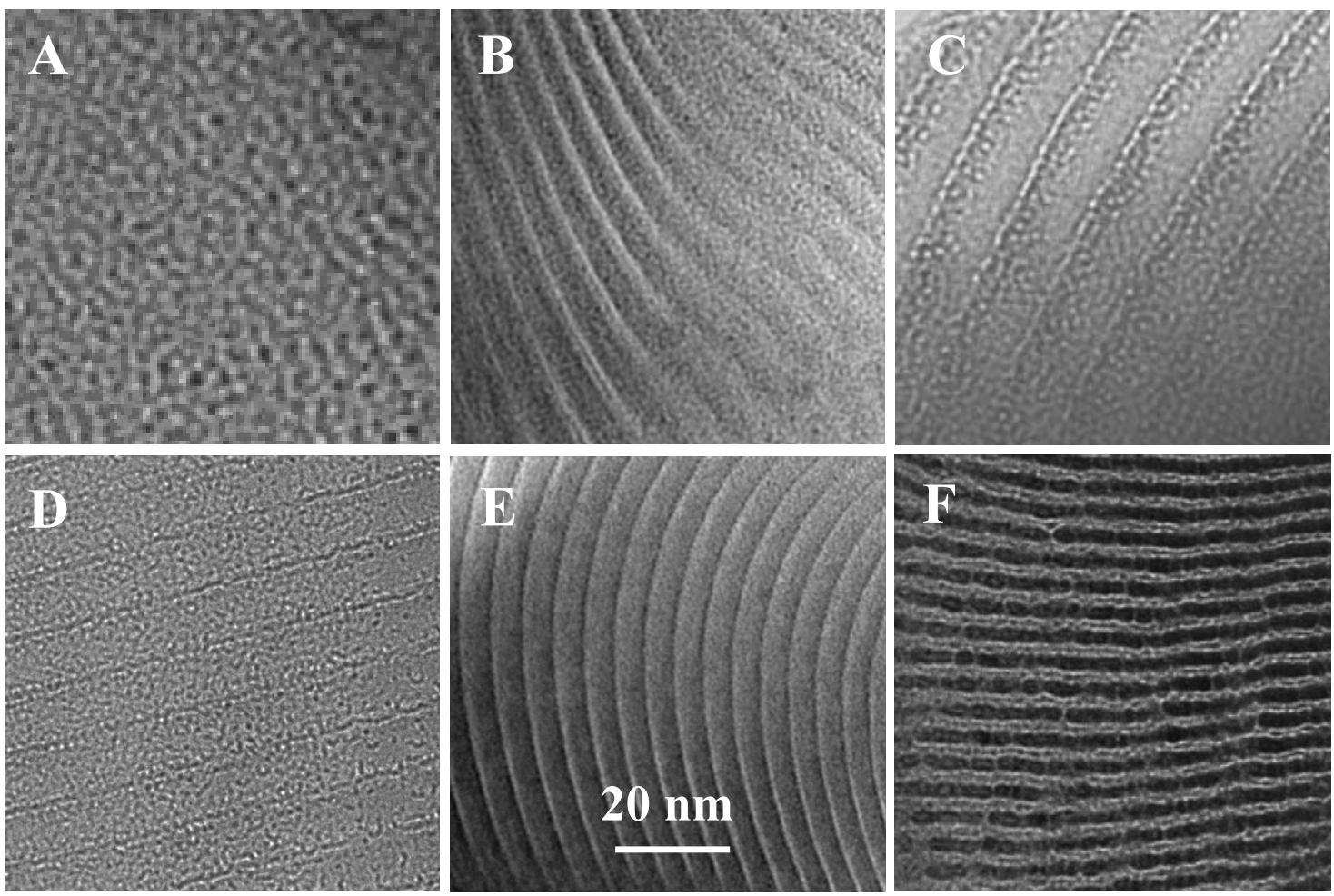

Fig. 1 TEM images of a polymer film irradiated by $4.2 \mathrm{keV} \mathrm{Ar}^{+}$ions at an angle of $80^{\circ}$ from normal direction with different ion fluences. A: before irradiation; B: $10^{17}$ ions $/ \mathrm{cm}^{-2} ; \mathrm{C}: 4 \times 10^{17}$ ions $/ \mathrm{cm}^{-2}$; D: $6 \times 10^{17}$ ions $/ \mathrm{cm}^{-2}$; E and $\mathrm{F}$ shows a curved and a smaller ripple structure with a $7 \mathrm{~nm}$ wavelength.
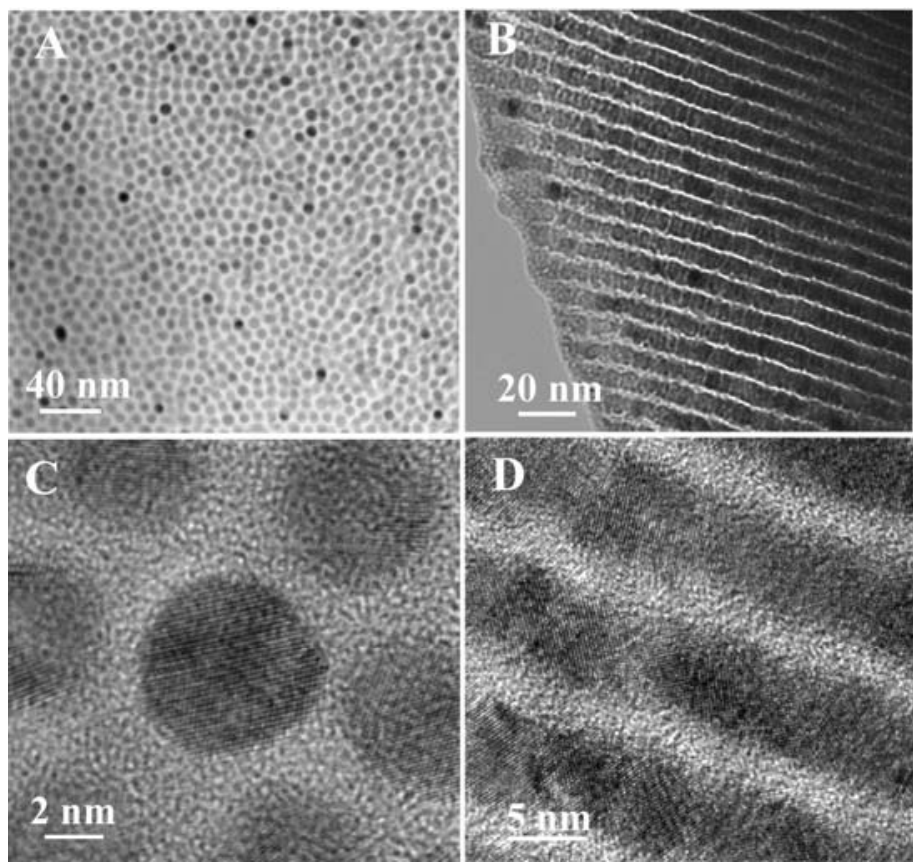

Fig. 2 TEM images of copper nanocrystal patterns on the polymer film due to ion beam sputtering and redeposition in an ion mill. A: uniformly distributed copper nano-dots; B: stripe-like nanostructure; $C$ : high resolution image of copper dots in A showing $7 \mathrm{~nm}$ in diameter and $2 \mathrm{~nm}$ spacing; D: high resolution image of an area in $\mathrm{B}$. 